\title{
Taxi Model of Unmanned Aerial Vehicle: Formulation and Simulation
}

\author{
Maryam Wasim ${ }^{1}$, Mukhtar Ullah ${ }^{1}$, Jamshed Iqbal ${ }^{1,2, *}$ \\ ${ }^{1}$ Department of Electrical Engineering, FAST National University of Computer and Emerging Sciences, \\ Islamabad, Pakistan \\ ${ }^{2}$ Electrical and Computer Engineering Department, University of Jeddah, Saudi Arabia \\ [maryam.wasim, mukhtar.ullah, *jamshed.iqbal]@ nu.edu.pk
}

\begin{abstract}
Unmanned Aerial Vehicles cannot work efficiently without a control system. Before any form of control is applied to it, modeling of the system is necessary. This work focuses on the development of a Taxi model of UAV. The reformulated model has been developed and simulated in Simulink and Simscape. Three scenarios have been considered in Simulation: Normal conditions with no wind, head and tail wind of $15 \mathrm{~m} / \mathrm{s}$ and steering angle of 1 radian. The key objective of this model is to include all the necessary dynamics of UAV when it is in taxi phase. This has been done by modifying the vehicle body model in Simscape to fit to the specifications of the UAV and moreover integrating it with an undercarriage model from previous literature to include the forces.
\end{abstract}

\section{Keywords-Unmanned Aerial Vehicle; Taxi model; Takeoff}

\section{INTRODUCTION}

UAVs play a vital role in defense and military operations. They carry out many vital and sensitive tasks in all kinds of environmental conditions, therefore it is essential that the performance of UAV does not fall when it is subjected to external disturbances. To ensure this, a control strategy has to be developed but before control comes modeling. If the model of UAV is not complete and accurate, any control strategy developed would fail.

The takeoff of a UAV is divided into four different phases. As shown in Fig. 1 that the takeoff starts with the 'taxi phase' and then proceeds to the 'acceleration phase' and 'rotation phase'. This is finally followed by the 'climb out phase'.

\begin{tabular}{|c|c|c|c|} 
Phase 1 & Phase 2 & Phase 3 & Phase 4 \\
\hline Taxi phase & Acceleration phase & Rotation phase & Climb out phase
\end{tabular}

Fig. 1. Phases of UAV takeoff

According to the different positions of the UAV on the runway during takeoff, the requirements of speed changes accordingly. Table I summarizes the requirements corresponding to each phase. The focus of this work is only on the first part that is the Taxi phase. During the taxi phase, a UAV has very low speed and the primary requirement is to keep the vehicle on the centerline. Control strategies ensure that the UAV does not deviate from the center because otherwise it will take off in the wrong direction.

All three wheels of the UAV remain on the ground during the taxi phase. So, the model derivation of UAV should include ground dynamics. As the speed of the UAV is very low during the taxi phase, the aerodynamic forces do not have a very strong effect on the UAV.

TABLE I

REQUIREMENT OF UAV TAKEOFF

\begin{tabular}{|l|l|}
\hline \multicolumn{1}{|c|}{$\begin{array}{c}\text { Phases of } \\
\text { UAV takeoff }\end{array}$} & \multicolumn{1}{c|}{ Requirement } \\
\hline Taxi & $\begin{array}{l}\text { Low speed. } \\
\text { Line up aircraft on runway. }\end{array}$ \\
\hline Acceleration & Accelerate to takeoff speed. \\
\hline Rotation & Rotate to make aircraft airborne. \\
\hline Climb Out & $\begin{array}{l}\text { Gain altitude. } \\
\text { Constant airspeed. }\end{array}$ \\
\hline
\end{tabular}

\section{LITERATURE REVIEW}

In scientific literature, there are many reported models of UAV which cover the ground dynamics and flight dynamics. Moreover it also reports many types of UAVs for example quad rotor and three-wheeled UAV. However, keeping in view the subject matter, the literature review here focuses only on the taxi model of three-wheeled UAVs.

In [1], Yang and $\mathrm{Wu}$ have developed a ground-taxiing model and proposed a control scheme for taxiing and taking off for a three-wheeled UAV. A nonlinear model for the taxiing phase has been developed by using the mathematical model but the transient values are not discussed. They have used stress analysis to incorporate the transients in the design. It is important to note that aerodynamic forces while the UAV is in taxi phase has not been included in the model. For a complete and realistic model, these forces should be included.

Automatic Take Off and Landing (ATOL) systems are very useful for UAVs as mentioned in [2]. The modeling of these vehicles are necessary if you want to control their motion. A fixed wing UAV has been considered for this system and the objective of this research was to develop a mathematical model for the ground run phase of a lightweight UAV. Based on an existing approach, the ground run phase is divided into different 
portions in order to make control design more efficient for each phase. After deriving equations of motions for the aircraft, the model is developed in Simulink for simulations and analysis. Undercarriage forces and moments are given in (1) and (2).

$$
\begin{gathered}
{\left[\begin{array}{l}
X_{u} \\
Y_{u} \\
Z_{u}
\end{array}\right]_{B}=\left[\begin{array}{c}
X_{s} \cos \left(-\delta_{s}\right)-Y_{s} \sin \left(-\delta_{s}\right)+X_{l}+X_{r} \\
X_{s} \sin \left(-\delta_{s}\right)+Y_{s} \cos \left(-\delta_{s}\right)+Y_{l}+Y_{r} \\
-N_{s}-N_{l}-N_{r}
\end{array}\right]} \\
{\left[\begin{array}{c}
L_{u} \\
M_{u} \\
N_{u}
\end{array}\right]_{B}=\left[\begin{array}{c}
-l_{l} Z_{l}+l_{r} Z_{r} \\
-l_{s} Z_{s}+l_{m} Z_{l}+l_{m} Z_{r} \\
l_{s} Y_{s}+l_{l} X_{l}-l_{m} Y_{l}-l_{r} X_{r}-l_{m} Y_{r}
\end{array}\right]_{B}}
\end{gathered}
$$

where, $X_{u}, Y_{u}, Z_{u}$ are the undercarriage forces while $L_{u}, M_{u}, N_{u}$ are the undercarriage moments. $\delta_{s}$ is the steering angle. Subscripts ' 'l', 'r' and's' are used for left rear wheel, right rear wheel and steering wheel respectively. Subscript B denotes the body axis system. Simulation results validated the mathematical model. The 6 DOF equations of motion contain undercarriage, thrust and gravity forces and moments. A small UAV with $22 \mathrm{~kg}$ mass, and $100 \mathrm{~N}$ thrust is used for simulation. However, no field testing has been done and therefore the values of friction and wind coefficients are not accurate and can affect the model. Also the aerodynamic forces have been completely ignored as velocity of the vehicle is considered to be low.

In [3], the research conducted on ATOL algorithms for a fixed wing UAV has been presented. The nonlinear runway model was developed considering aerodynamic, engine and gravitational effects based on research reported earlier. Runway forces and moments, that are a result of an interaction of the aircraft's landing gear and ground, were calculated to develop a comprehensive model. Runway forces include friction forces and cornering forces given by (3) and (4) respectively.

$$
\begin{aligned}
& F_{f}=\mu_{f} N \\
& F_{c}=C_{\alpha} \alpha=C_{\alpha \alpha} \alpha
\end{aligned}
$$

where, side slip angle $\alpha$ is the angle between the direction of travel and the wheel's heading direction. $\mu_{\mathrm{f}}$ is the coefficient of rolling resistance and $C_{\alpha \alpha}$ is the tire's cornering coefficient. The non-linear model is then linearized by assuming that the roll and pitch angles are zero on the runway and all the aerodynamic and runway forces that contribute to yaw moment can be ignored.

In [4] nonlinear model is reported for a UAV's ground taxiing phase by using both kinematic and dynamic analysis of the system. An automatic system to control the UAV motion is then developed. Following the formation of an axis system, the computed forces on the UAV include aerodynamic forces, gravity forces, power of the engine, support forces of the landing gear, friction forces and side forces. The 6 DOF system is reduced to 3 DOF when it is in the ground taxiing phase. Simulation results proved that the model developed was very accurate but at the same time it is also very complex.

Dynamic modeling has been carried out in [5] to obtain the ground taxiing model of UAV. The forces and moments acting on UAV that result from ground have been discussed in detail. These characteristics include frictional force, supporting force along with the moment produced by these forces. Supporting forces are obtained by using the mechanical properties of tires such as the compression of tires and landing gears and their compression rate. Both of them are considered as linear discrete systems with constant stiffness and damping. The lateral friction and rolling friction have been calculated and added to the model. The final model is completed by adding gravity, aerodynamic and thrust from piston propeller engine. The model is then linearized using small perturbation theory and further work has been done on the design of its controller. Another model for taxiing has been developed in [6]. The forces and moments used in this model are the lateral friction of the wheels and support forces from ground to the nose gear. To simplify the computations, the wheels and the struts are assumed to be rigid. Also the aerodynamic forces are considered to be linear in the model and the simulation is terminated when the sideslip angle goes beyond a certain threshold value.

In contrast to several reported works, it is not practical to assume the tires as rigid bodies. When it comes to the establishment of tire road interaction, one of the most renowned works has been reported by [7]. The formula derived is known as the 'Magic Formula' and is used to model the longitudinal forces that arise due to tire-ground interaction. Results from this work has been applied to an Unmanned Ground Vehicle (UGV) in [8] and the longitudinal and lateral forces on the tires are calculated using this proposed magic formula. The same formula has also reported in [9] for modelling the wheel.

The model reported in [10] for control of aircraft during runway acceleration, in the presence of winds, is the same as [11]. The model has the undercarriage forces and aerodynamics forces. The model parameters have been fitted to the characteristics of Boeing-727. The model used by [12] is very complex and has very strong nonlinearity as it includes the aerodynamic model, ground taxiing model, the deviation correction model and moreover the antiskid model.

Another method for modeling of UAV has been done by Triputra et al. in [13]. It uses the DATCOM+ software to calculate the different aerodynamic coefficients. Additionally MATLAB identification toolbox has been used to form empirical linear state-space model based on flight test data. There are two methods for identification, blackbox method and grey-box method. Grey-box method uses both physical principles and measurement data whereas balckbox method relies only on measurement data. Both of these methods have been implemented to build a state-space model for the UAV. The downside is that this form of linear state space model can only work for a certain altitude and speed. This work has been further improved in [14] by developing a nonlinear model. The aerodynamic coefficients can be calculated as a function of airspeed and altitude. A 10 DOF differential equations in the form of feedback systems have been used to represent a nonlinear dynamic model. Simulations have been carried out to verify the nonlinear model. In another work the same model reported in [13] has been used by [15] to improve its computational speed. This has been accomplished by performing all of the UAV model computations using $\mathrm{C}$ language instead of MATLAB. It is also verified that the output of $\mathrm{C}$ program has very less error. Collision avoidance of Autonomous Vehicles (AVs) has been discussed in [16]. A mathematical model, inspired by Richardson's arms race model, 
has been used to imitate the mirroring and mentalizing function of the human brain. The simulation results showed that the proposed strategy is $78.52 \%$ more efficient than the existing random walk based strategy. In [17], the collision avoidance of AVs has been improved by introducing human social norms and emotions in the AV's autopilot so that they can act just like wellbehaved drivers. Each AV has been given a different personality and a society of AVs has been formulated and simulated. The results demonstrated over-performance of this method compared to random walk based strategy.

The literature suggests that for the modeling of taxi phase, the ground dynamics and the forces resulting from road and tire interaction should definitely be included. The aerodynamics has been ignored in some reported cases for modeling of the taxi phase since the speed of the UAV is considered to be low during this phase.

\section{METHODOLOGY}

Modeling of a system finds motivation to design control law $[18,19]$. During taxi phase, all the three wheels of the UAV stay on the ground and therefore the UAV can be modelled as a ground vehicle. After going through various models presented in the literature, two of them are combined using Simscape and Simulink. The two selected models are the 'vehicle body model' and the 'undercarriage model'.

\section{A. Vehicle Body Model}

The vehicle body used in the present work is taken from Simscape. The model as shown in Fig. 2 is used to represent a vehicle body in SIMSCAPE. Table II presents the nomenclature of the variables used in the model.

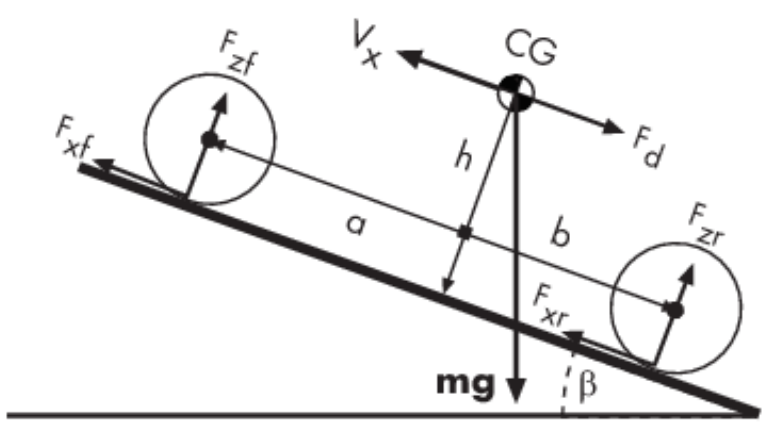

Fig. 2. Vehicle body model from Simscape

Equations (1-5), represent the vehicle body model. Zero normal acceleration and zero pitch torque determine the normal force on each of the front and rear wheel. The wheel normal forces must satisfy (6).

TABLE II

VEHICLE MODEL VARIABLES

\begin{tabular}{|l|l|}
\hline Symbol & \multicolumn{1}{c|}{ Description } \\
\hline$g$ & Gravitational acceleration \\
\hline$\beta$ & Angle of inclination \\
\hline$m$ & Vehicle's mass \\
\hline$h$ & Height of vehicle center of gravity above the ground \\
\hline$a, b$ & $\begin{array}{l}\text { Distance of front and rear axle, respectively from } \\
\text { the vehicle's center of gravity }\end{array}$ \\
\hline
\end{tabular}

\begin{tabular}{|l|l|}
\hline$V_{X}$ & $\begin{array}{l}\text { Velocity of the vehicle. Velocity is positive in } \\
\text { forward direction. }\end{array}$ \\
\hline$V_{W}$ & Wind speed. Positive when headwind. \\
\hline$n$ & Number of wheels on each axle \\
\hline$F_{x f}, F_{x r}$ & Longitudinal forces on each wheel. \\
\hline$F_{z f}, F_{z r}$ & Normal load forces on each wheel \\
\hline$A$ & vehicle frontal cross-sectional area \\
\hline$C_{d}$ & Aerodynamic drag coefficient \\
\hline$\rho$ & Mass density of air \\
\hline$F_{d}$ & Aerodynamic drag force \\
\hline
\end{tabular}

$$
\begin{aligned}
& m \dot{V}_{x}=F_{x}-F_{d}-m g \cdot \sin \beta \\
& F_{x}=n\left(F_{x f}+F_{x r}\right) \\
& F_{d}=\frac{1}{2} C_{d} \rho A\left(V_{x}+V_{w}\right)^{2} \cdot \operatorname{sgn}\left(V_{x}+V_{w}\right) \\
& F_{z f}=\frac{-h\left(F_{d}+m g \sin \beta+m \dot{V}_{x}\right)+b \cdot m g \cos \beta}{n(a+b)} \\
& F_{z r}=\frac{+h\left(F_{d}+m g \sin \beta+m \dot{V}_{x}\right)+a \cdot m g \cos \beta}{n(a+b)} \\
& F_{z f}+F_{z r}=m g \cdot \cos \beta / n
\end{aligned}
$$

This vehicle body model can be adjusted to represent a UAV. This is done by giving the parameters the desired values and also by considering the front axle single wheel instead of two. However there are many other forces associated with the taxi model of a UAV and the model of Simscape does not include these forces. Therefore, an undercarriage model reported in literature [11] and combined with this model in Simscape so as to make the model more efficient.

\section{B. Undercarriage Model}

The undercarriage model of a UAV has all the important forces that a UAV experiences while taxiing on the runway [11] . Higher level block diagram of undercarriage model is shown in Fig. 3. Both the yaw rate dynamics and sideslip dynamics are included in the model. These forces actually determine the UAV's movement on the runway. The normal force $\mathrm{N}$ and $\mathrm{U}$ (equal to $\mathrm{V}_{\mathrm{x}}$ ) are coming from the vehicle body model. $\delta_{\mathrm{s}}$ is the steering angle and $\mathrm{B}$ is the yaw angle.

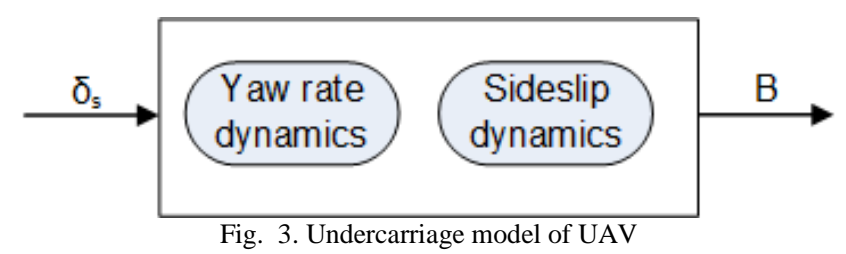

This undercarriage model has been implemented using Simulink and has been combined with the vehicle body model in Simscape mentioned before. This model adds more details to the vehicle body model by adding all the necessary 
undercarriage forces. Fig. 4 shows the Simulink and Simscape diagram for the reformulated taxi model of UAV. The steering angle $\delta_{\mathrm{s}}$ is given to UAV to check its effect on yaw angle of the UAV. The velocity and distance covered by UAV are also measured. The vehicle body block contains the vehicle body model implemented in Simscape. As the UAV under consideration is three wheeled, therefore it has two rear wheels and one front wheel detailed in Fig. 5. This vehicle body uses the magic formula given in [7] to model the behavior of its tires. The 'motion sensor' is used to detect the motion of the UAV and gives the velocity and distance covered by the UAV. The PSSimulink (Physical signal to Simulink) is used to plot the physical signals using scope in Simulink. The undercarriage forces are implemented in Simulink and to combine it with the vehicle body the normal force and velocity of UAV are taken from it. The yaw angle is computed using the model reported in [11] and then the results are shown as a plot in Simulink

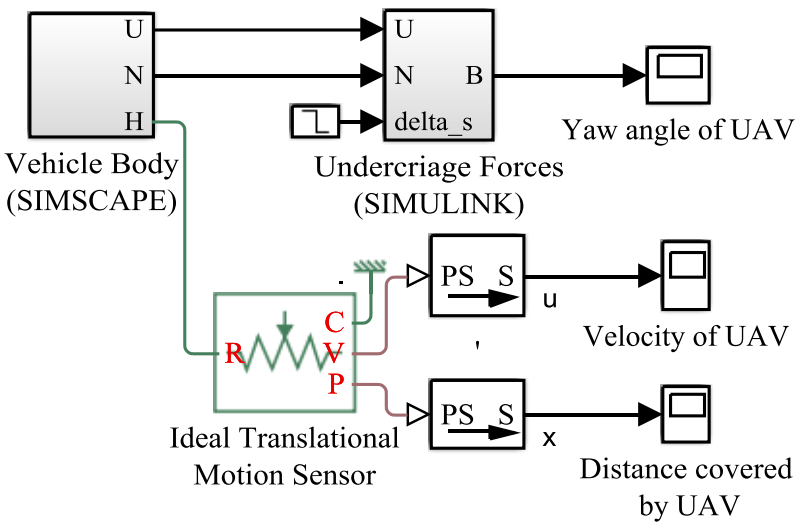

Fig. 4. Reformulated taxi model of UAV

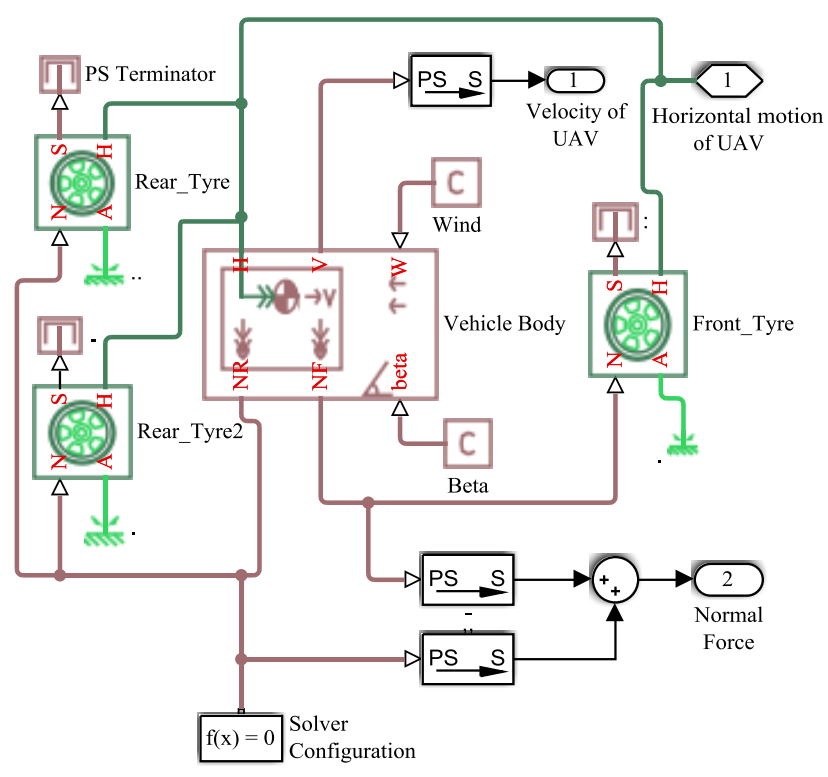

Fig. 5. Vehicle body model in Simscape

\section{RESULTS}

A basic UAV taxi model with undercarriage forces is reformed using Simulink and Simscape. The different scenarios in which the UAV model has been simulated are mentioned below:

\section{A. Output of UAV with no wind disturbance}

When the UAV is operating under normal conditions, it will not be subjected to any form of wind disturbance. The angle of inclination $\beta$ as shown in Fig. 2, is given as -2 radians, hence the UAV is actually moving down the slope. The velocity and distance covered by the UAV is shown in Fig. 6 and Fig. 7 respectively. The resistive drag force and friction force is not enough to balance the downward force, causing an increase in the velocity and distance covered by the vehicle.

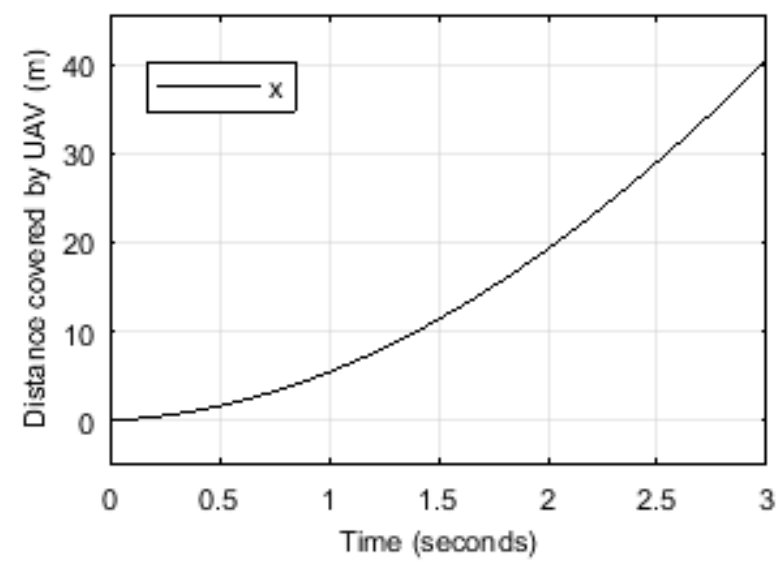

Fig. 6. Distance covered by UAV in no wind

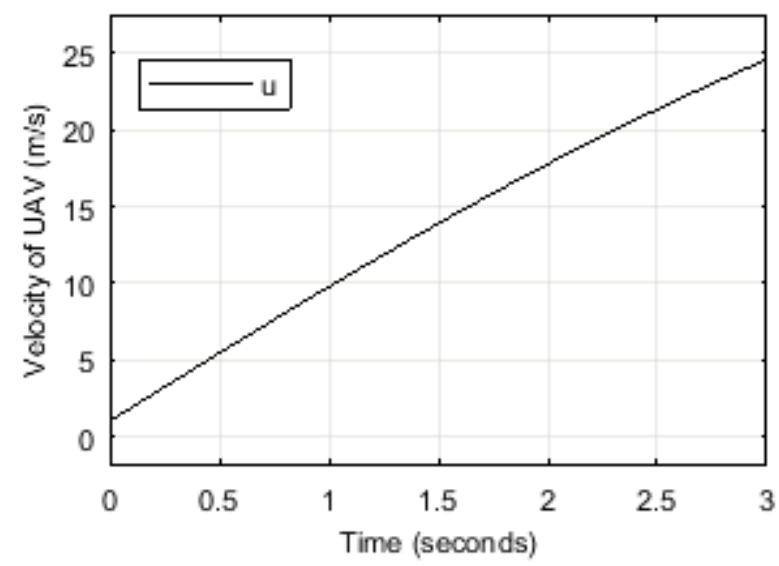

Fig. 7. Velocity of UAV in no wind

\section{B. Output of UAV with headwind and tailwind}

The vehicle body model has some aerodynamics included in the form of drag force, the UAV should experience some change in motion when it encounters a headwind and tailwind. The simulation scenario is the same as in previous part first with headwind of $15 \mathrm{~m} / \mathrm{s}$ included and the results are shown in Fig. 8 and Fig. 9. The case of tailwind is also considered and the results illustrated in Fig. 10 and Fig. 11. As the headwind is in the 
opposite direction to the motion of UAV, therefore the velocity of the UAV is less than in the case of no wind. The tailwind is in the same direction as the motion of UAV. When we apply tail wind to UAV it gets a supporting force and the velocity increases and hence more distance is covered by the UAV.

The simulation has been carried out for 3 seconds. In case of no wind, the maximum velocity acquired by the UAV is $24 \mathrm{~m} / \mathrm{s}$ and the distance covered during this time is 40 meters. Whereas in the case of headwind, which opposes the UAV motion, the UAV acquires a maximum velocity of $17 \mathrm{~m} / \mathrm{s}$ and the distance covered is 30 meters. Finally, in case of tailwind, the UAV attains a speed of $27 \mathrm{~m} / \mathrm{s}$ with 42 meters as the distance covered.

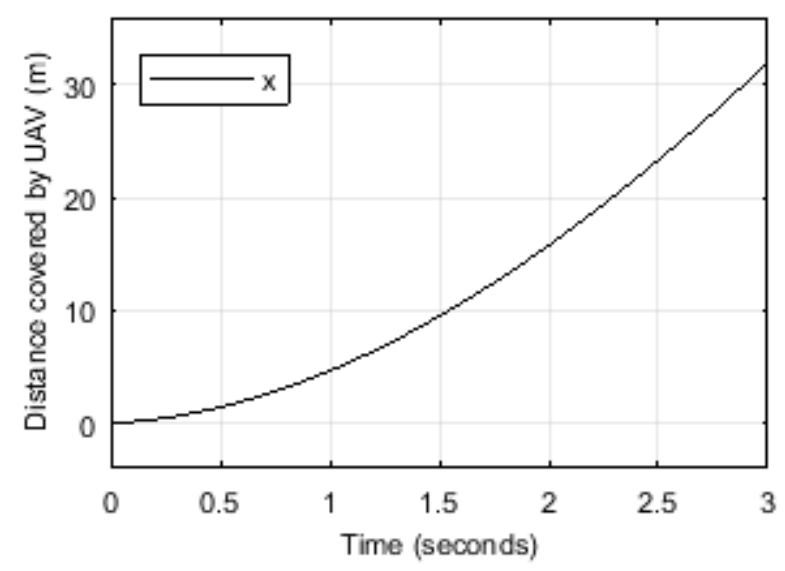

Fig. 8. Distance covered by UAV with headwind $15 \mathrm{~m} / \mathrm{s}$

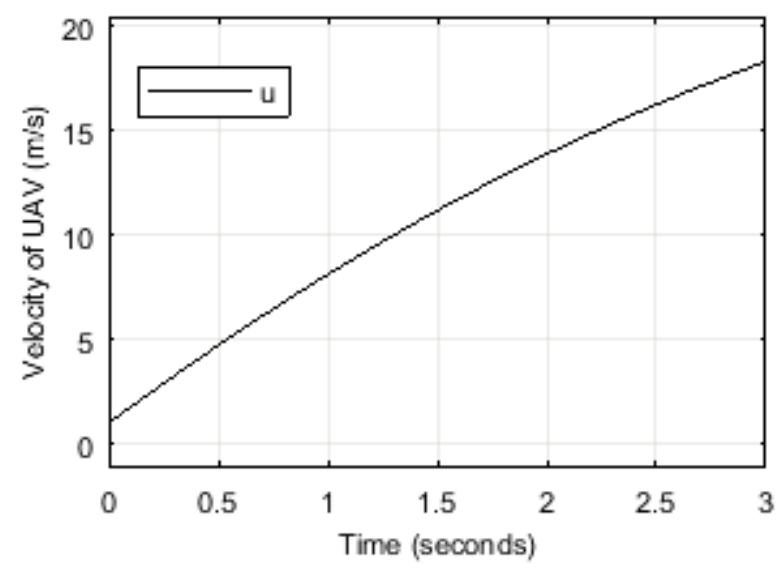

Fig. 9. Velocity of UAV with headwind $15 \mathrm{~m} / \mathrm{s}$

\section{Effect on Yaw Angle without steering angle}

The undercarriage model of UAV is driven by the velocity of UAV from vehicle body model, the normal force and a steering angle. The steering angle controls the yaw angle of the UAV. As the UAV is going down the centerline of the runway, the steering angle can be used to keep the UAV on track. Three different scenarios are discussed with yaw angle of UAV. Firstly, the UAV is given no steering angle to see if it deviates from the centerline, but as the UAV does not experience any other disturbance, it keeps on track as shown in Fig. 12.
Secondly a unit step is provided at the steering angle to see its effect. As expected the UAV changes its direction and its yaw angle starts to increase. Thirdly, the UAV is tested with a negative step at steering angle and the UAV goes in the opposite direction since the yaw angle now starts to decrease.

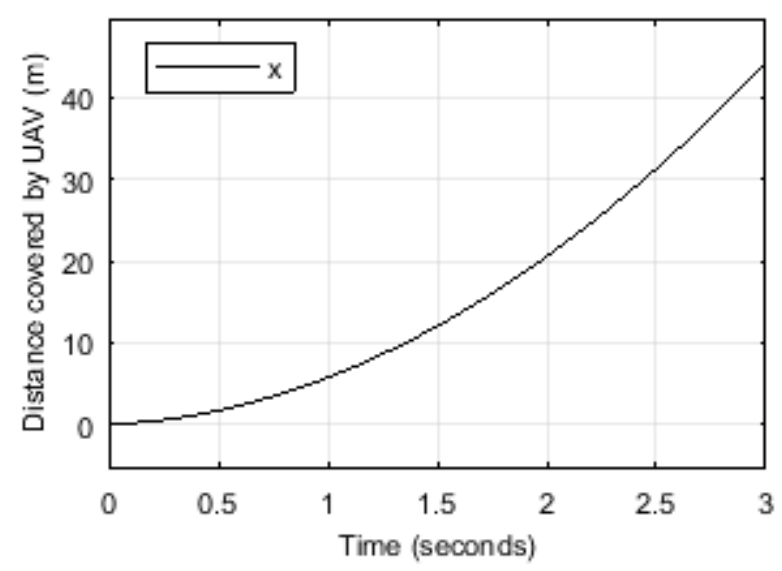

Fig. 10. Distance covered by UAV with tailwind $15 \mathrm{~m} / \mathrm{s}$

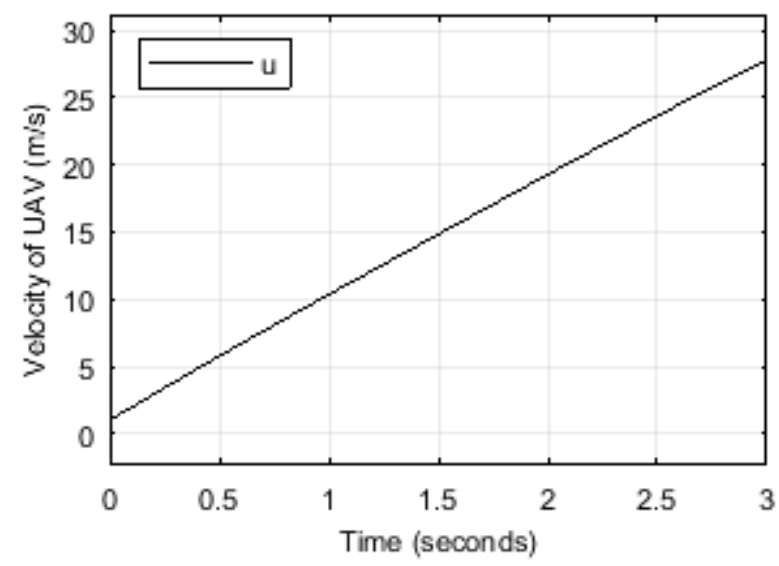

Fig. 11. Velocity of UAV with tailwind $15 \mathrm{~m} / \mathrm{s}$

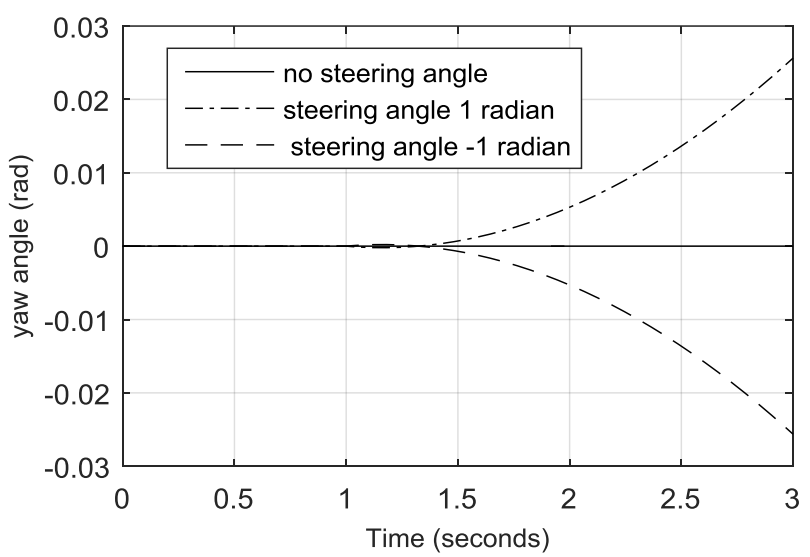

Fig. 12. Behavior of yaw angle with changes in steering angle 


\section{CONCLUSION}

UAVs have become very popular in recent times. Automatic takeoff and landing of UAV is a challenging problem which is popular in control and robotics community. This work focuses on the modeling of the taxi phase of UAV. The model has been reformulated by combining two models together that is the 'vehicle body model' in Simscape and the 'undercarriage model' in Simulink. Simulation results are presented illustrating UAV working without wind and in the presence of headwind and tailwind. Moreover, the yaw angle of UAV also changes when the UAV is given a steering angle. This work can be extended to develop a more in depth model of UAV. The aerodynamics of the UAV can be included in a more elaborated form. Moreover, some form of control strategy can also be applied to the UAV to keep it on the centerline in case of any external disturbance.

\section{REFERENCES}

B. Yan and C. Wu, "Research on taxi modeling and taking-off control for UAV," in Computational Intelligence and Design (ISCID), Seventh International Symposium on, 2014, pp. 108-111. M. Essuri, K. Alkurmaji, and A. Ghmmam, "Developing a dynamic model for unmanned aerial vehicle motion on ground during takeoff phase," in Applied Mechanics and Materials, 2012, pp. 561-567. J.-C. Roos and I. Peddle, "Autonomous take-off and landing of a low cost unmanned aerial vehicle," $R \& D$ Journal of the South African Institution of Mechanical Engineering, vol. 25, 2009.

[4] H. Song and X. Chen, "Model and auto take-off control law design of unmanned aerial vehicle," in Advanced Materials Research, 2012, pp. 1524-1530.

[5] J. Gao and H. Jia, "Control research for a small fixed-wing UAV during ground taxiing," Journal of Harbin Institute of Technology, vol. 2, pp. 8-13, 2017

[6] L. Song, H. Yang, X. Yan, C. Ma, and J. Huang, "A study of instability in a miniature flying-wing aircraft in high-speed taxi," Chinese Journal of Aeronautics, vol. 28, pp. 749-756, 2015. H. Pacejka, Tire and vehicle dynamics, Second ed.: Elsevier, 2005.

[8] J. Han, G. Ren, D. Li, and Z. Jia, "Turning model of a skid-steering unmanned ground vehicle under steady state conditions based on magic formula," International Conference on Mechatronics Engineering and Information Technology, vol. 57, pp. 9-15, 2016.
[9] R. O. Kuchar, "A versatile Simulation environment for Design Verification, System Integration Testing and Pilot Training of a diamond-shaped Unmanned Aerial Vehicle," in 2018 AIAA Modeling and Simulation Technologies Conference, 2018, p. 0126.

[10] N. Botkin and V. Turova, "Aircraft runway acceleration in the presence of severe wind gusts," in IFIP Conference on System Modeling and Optimization, 2015, pp. 147-158.

[11] R. D. De Hart, "Advanced take-off and flight control algorithms for fixed wing unmanned aerial vehicles," Doctoral dissertation, Stellenbosch: University of Stellenbosch, 2010.

[12] S. Dong, Z. Jiao, X. Sun, and X. Liu, "Dynamic allocation algorithm for the gain of UAV nose wheel steering and differential braking based on decomposition control," in Aircraft Utility Systems (AUS), IEEE International Conference on, 2016, pp. 831-835.

[13] F. R. Triputra, B. R. Trilaksono, R. A. Sasongko, and M. Dahsyat, "Longitudinal dynamic system modeling of a fixed-wing UAV towards autonomous flight control system development: A case study of BPPT wulung UAV platform," in System Engineering and Technology (ICSET), International Conference on, 2012, pp. 1-6.

[14] F. R. Triputra, B. R. Trilaksono, T. Adiono, R. A. Sasongko, and M. Dahsyat, "Nonlinear dynamic modeling of a fixed-wing unmanned aerial vehicle: A case study of Wulung," Journal of Mechatronics, Electrical Power, and Vehicular Technology, vol. 6, pp. 19-30, 2015.

[15] Y. A. Prabowo, B. R. Trilaksono, and F. R. Triputra, "Hardware inthe-loop simulation for visual servoing of fixed wing UAV," in Electrical Engineering and Informatics (ICEEI), International Conference on, 2015, pp. 247-252.

[16] F. Riaz and M. A. Niazi, "Towards social autonomous vehicles: Efficient collision avoidance scheme using Richardson's arms race model," PloS one, vol. 12, p. e0186103, 2017.

[17] F. Riaz, S. Jabbar, M. Sajid, M. Ahmad, K. Naseer, and N. Ali, "A collision avoidance scheme for autonomous vehicles inspired by human social norms," Computers \& Electrical Engineering, 2018.

[18] W. Alam, A. Mehmood, K. Ali, U. Javaid, S. Alharbi and J. Iqbal, "Nonlinear control of a flexible joint robotic manipulator with experimental validation", Strojniški vestnik - Journal of Mechanical Engineering, vol. 64, pp. 47-55, 2018.

[19] S. A. Ajwad, J. Iqbal, R. U. Islam, A. Alsheikhy, A. Almeshal and A. Mehmood, "Optimal and robust control of multi DOF robotic manipulator: Design and hardware realization", Cybernetics and Systems, vol. 49, pp. 77-93, 2018. 